\title{
On spacetime duality and the Astromechanics of a dual Universe
}

\author{
Mohammed. B. Al-Fadhli ${ }^{*}$ \\ ${ }^{1}$ College of Science, University of Lincoln, Lincoln, LN6 7TS, UK. \\ *Correspondence: malfadhli@lincoln.ac.uk; mo.fadhli7@gmail.com
}

\begin{abstract}
Recent astronomical measurements of the fine structure constant revealed it varies slightly through specific directions, which could indicate a directional/anisotropic universe. A curvature in complex spacetime can be interpreted as spatial warping evolution along with its travel through the imaginary time dimension. Complex spacetime worldlines of the universe spatial factor evolution through the imaginary time are utilised to construct a potential cosmic topology. The worldlines of a positively curved universe governed by gravity alone revealed $\mp$ solutions, which imply that the matter and antimatter could be evolving in opposite directions as distinct universe sides; potentially corroborating the axis of the cosmic microwave background and the directional universe. The model indicates a phase of decelerating spatial expansion during the first $\sim 9 \mathrm{Gyr}$, which is followed by a second phase of accelerating expansion; theoretically resolving the tension in the Hubble parameter measurements, with predicted density at the phase transition of $\Omega_{t}=\sim 1.12>1$. Additionally, it predicts a final time-reversal phase of spatial contraction leading to a Big Crunch, signalling a cyclic universe. On the spacetime duality, simulations of the spacetime continuum flux along with its predicted worldlines demonstrated the fast-orbital speed of stars due to an external momentum exerted on galaxies via curvature through the imaginary time dimension. These findings indicate the antimatter can exist as a distinct side, which influences the evolution of the universe.
\end{abstract}

Keywords: Accelerated expansion, fast-rotation, flatness, parallel universe, duality, antimatter

\section{INTRODUCTION}

Advances in cosmology and astronomical observations have revealed numerous inconsistencies with our traditional view of the universe. Recent astronomical measurements in four directions of the fine structure constant by several studies discovered it varies slightly through two directions only, which could indicate an anisotropic universe. However, the variation is not depending on time only but occurs as well through specific directions [1-3]. Therefore, the perception of constant speed of light in the vacuum throughout the universe evolution could be a good approximation to reality.

Although no rigorously tested theories describing a potential cosmic topology currently exist, it might be feasible to be constructed utilising complex spacetime worldlines of the universe spatial factor evolution through imaginary time; where a curvature in complex spacetime can be clarified as spatial warping evolution along with its travel through imaginary time dimension. Cosmic topology, i.e., universal spacetime geometry could demonstrate the movement of matter over the evolution of the universe and may explain the anisotropic universe and other universe's unsolved problems.

Additionally, bounce cosmology could provide an agreement with anisotropy observations in the cosmic microwave background (CMB) such as the potential early universe positive curvature [4], $\mathrm{CMB}$ axis and dipole anisotropy. The non-singular Big Bounce theory assumes that the primordial substance was concentrated from a previous collapsed universe, with the universe experiencing continuous expansions and contractions; where the initial spatial factor is greater than zero $[5,6]$. However, another version of the theory could be postulated that a Bounce Bang of the primordial substance at thermal equilibrium produced a hot and dense early universe where the matter and antimatter could have been separated by the electromagnetic fields $[7,8]$ and propelled in opposite directions in corroboration to the $\mathrm{CMB}$ dipole anisotropy. 
Recently, the current $\Lambda$ cold dark matter model ( $\Lambda$ CDM) [9] has faced inconsistencies by the advancement of new astronomical observations [4,10]. The recent Planck Legacy 2018 (PL18) release indicated the existence of an enhanced lensing amplitude in the CMB from the early universe that is higher than what is expected in the $\Lambda \mathrm{CDM}$ [11], which endorses the existence of a positive curvature with a confidence level greater than 99\% [4]. Additionally, the observed gravitational lensing by substructures of 11 galaxy clusters is more than an order of magnitude of what predicted by the $\Lambda \mathrm{CDM}$ [12]. Moreover, the precise Hubble parameter measurements from the early universe, using the Planck datasets based on the $\mathrm{CMB}$, showed a lower expansion rate value in comparison with the value of the Hubble parameter in the present universe using the type Ia supernovae distance-redshift method [10,13-15], where the variation is three standard deviations [4,10]. Riess in 2020 found the expansion of the universe is faster than what $\Lambda \mathrm{CDM}$ estimates where the disagreement between several independent measurements taken from the early and present universe is at four to six-sigma [16]. Further, the expanding speed was found to change depending on the direction [17]. Thus, a profound adjustment of the $\Lambda \mathrm{CDM}$ or new physics are now growing due to this evidence underlying the model assumptions [18].

In this paper, the evolution of complex spacetime worldlines is utilised to construct a potential cosmic topology. A closed finite universe of non-singular Bounce Bang creation is adopted as it could be aligned with the quantum entanglement, where the energy present at the Bang is preserved indefinitely; therefore, cosmic conservation ensures the total spin of a pair of particles to be always conserved regardless of their locations [19]; and could provide an agreement with CMB observations [20]. The paper is organised as follows: In Section 2, the metric tensor is modified to account for the initial space curvature and the model is derived without a cosmological constant as its predicted value contradicts the quantum field theory [21]. Sections 3, 4 and 5 discuss the evolution of spacetime worldlines and the Hubble parameter. Section 6 demonstrates the fast-orbital star speed. Section 7 summarises the conclusions. Finally, Section 8 suggests future developments for this work.

\section{MATHEMATICAL MODEL}

The fine-structure constant was detected to vary through space and time [1-3], where the speed of light in vacuum, Newton's gravitational constant and other constants could be not constants throughout the evolution of the universe and its geometry. As an alternative, spacetime is postulated to have a dual nature that it curves according to general relativity as waves and flows as perfect fluid particles; where the spacetime modulus of elasticity $E_{D}$ is considered as a fundamental constant of nature in this work as follows

$$
c^{4}\left(t_{t \tau}\right)=8 \pi E_{D} G\left(t_{t \tau}\right)
$$

where $t_{t \tau}$ denotes a three-dimensional time tensor as the time could be abstractly considered as an anomaly over each spatial direction. $G$ and $c$ denote Newton's gravitational constant and the speed of light in vacuum regarding the time tensor respectively. The Einstein-Hilbert action could adapt the variations in these constants as

$$
S=\int\left[\frac{c^{4}\left(t_{t \tau}\right)}{16 \pi G\left(t_{t \tau}\right)} R+L\right] \sqrt{-g} d^{4} x
$$

where $R=R_{u v} g^{u v}$ denotes the scalar curvature regarding the three-dimensional space and the imaginary time component $\tau, R_{u v}$ is the Ricci curvature tensor, $g=\operatorname{det} g_{u v}$ is the determinant of the metric tensor $g_{u v}$ and $L$ represents the Lagrangian of matter/antimatter. The integration of this action over the whole spacetime using the principle of the least action yields Einstein's field equations without a cosmological constant while accounting for the variations in the constants (parameters)

$$
R_{u v}-\frac{1}{2} R g_{u v}=\frac{8 \pi G_{\alpha}}{c_{\alpha}{ }^{4}} T_{u v}
$$

where $T_{u v}$ is the energy-momentum tensor [22] and the parameters are denoted with regards to $t_{t \tau}$. 
Based on equivalent Newtonian dynamics, the isotropic spherical coordinates of the FriedmannLemaître-Robertson-Walker (FLRW) metric model [23,24] can be enhanced to account for the initial space curvature as well as to utilise the imaginary time component

$$
d s^{2}=-c_{\alpha}{ }^{2} d \tau^{2}-a^{2}(-i \tau)\left(\frac{d r^{2}}{1-k \frac{r^{2}}{r_{0}{ }^{2}}}+r^{2} d \theta^{2}+r^{2} \sin ^{2} \theta d \phi^{2}\right)
$$

where $d s$ is the four-dimensional complex spacetime interval in polar coordinates, $a$ is the spatial factor, $k$ and $r_{0}{ }^{2}$ are constants representing the space curvature and the initial space curvature, respectively. For a closed, open, and flat universe, $k=1,-1$, and 0 , while $r_{0}{ }^{2}=1, \gtrsim 1$, and $\lesssim 1$ for flat, positive and negative initial space curvatures respectively. The zero-zero component of the Ricci tensor and the scalar curvature are solved for the enhanced metric tensor as

$$
\begin{gathered}
R_{0}^{0}=-3 \frac{\ddot{a}}{a} \\
R=-\frac{1}{6 c_{\alpha}{ }^{2}}\left(\frac{\ddot{a}}{a}+\frac{\dot{a}^{2}}{a^{2}}+\frac{k c_{\alpha}{ }^{2}}{a^{2} r_{0}{ }^{2}}\right)
\end{gathered}
$$

where $a$ is a function of the imaginary time. By solving the field equations in Eq. (3) for a perfect fluid given by $T_{v}^{u}=\left(\rho+\frac{P}{c_{\alpha}^{2}}\right) u^{u} u_{v}+P \delta_{v}^{u}[22,25]$ and submitting Eqs. $(5,6)$ to them, Friedmann equations that count for the initial space curvature and variations in the parameters are obtained

$$
\begin{gathered}
H^{2} \equiv \frac{\dot{a}^{2}}{a^{2}}=\frac{8 \pi G_{\alpha} \rho}{3}-\frac{k c_{\alpha}{ }^{2}}{a^{2} r_{0}{ }^{2}} \\
\dot{H} \equiv \frac{\ddot{a}}{a}=-\frac{4 \pi G_{\alpha}}{3}\left(\rho+3 \frac{P}{c_{\alpha}{ }^{2}}\right)
\end{gathered}
$$

where $H, P$ and $\rho$ represent the Hubble parameter, pressure and density respectively. Friedman equations can be solved over the whole imaginary conformal time of one current universe life cycle, where $k=1$ by the definition of the FLRW metric for the positively curved universe. By rewriting Eq. (7) in terms of the imaginary conformal time in its parametric form $d \eta \equiv-i \frac{d \tau}{a}$, we get

$$
\int_{\eta_{0}}^{\eta} d \eta=\oint\left(\frac{8 \pi G_{\alpha} \rho_{0} a_{0}^{3}}{3} a-\frac{a^{2} c_{\alpha}^{2}}{r_{0}^{2}}\right)^{-\frac{1}{2}} d a
$$

where $\rho=\frac{\rho_{0} a_{0}{ }^{3}}{a^{3}}$ [26], $a_{0}$ is the spatial factor at the corresponding imaginary time $\tau_{0}$ and density $\rho_{0}$. By utilising Eq. (1) and then integrating, the evolution of the spatial factor is obtained

$$
a(\eta)=\frac{c_{\eta}^{2}}{12 E_{D}} \rho_{0} r_{0}^{2} a_{0}^{3}\left(1-\cos \frac{\eta}{r_{0}}\right)
$$

Additionally, the evolution of the universe imaginary time $\tau(\eta)$ can be obtained by integrating the length of the spatial factor contour of one universe life cycle over its expansion speed $H$, initiating at the imaginary time $\tau_{0}$ and the corresponding spatial factor $a_{0}$. Therefore, by rewriting Eq. (10) in terms of the Hubble parameter by its definition at imaginary time $\tau_{0}$ as follows $d \tau \equiv i \frac{d a(\eta)}{H a_{0}}$, we get

$$
\int_{\tau_{0}}^{\tau} d \tau=i \frac{c_{\eta}{ }^{2}}{12 E_{D} H} \rho_{0} r_{0}^{2} a_{0}^{2} \int_{\eta_{0}}^{\eta}\left(1-\cos \frac{\eta}{r_{0}}\right) d \eta
$$

By performing the integration, the evolution of the imaginary time is obtained

$$
\tau(\eta)=i \frac{c_{\eta}{ }^{2}}{12 E_{D} H} \rho_{0} r_{0}^{2} a_{0}^{2}\left(\eta-\sin \frac{\eta}{r_{0}}\right)+\tau_{0}
$$

where $\tau_{0}$ denotes the reference imaginary time for the temporal evolution. 
According to the non-singular Bounce Bang, the initial density of the primordial substance of the previous collapsed universe $\rho_{0}$ should have the highest value; then it should decrease due to the increase of universe size, where $a_{0}$ and $\tau_{0}$ are the corresponding initial spatial factor and imaginary time respectively. Therefore, according to the conservation of energy and because of the isotropy, the divergence of the energy-momentum tensor vanishes at $\Delta_{v} T^{u v}$ which yields $\frac{\dot{a}}{\mathrm{a}} T_{u}^{u}+3 \frac{\dot{a}}{\mathrm{a}} \rho-i \frac{\partial \rho}{\partial \tau}=0$ and $3(\rho+P) \frac{\dot{a}}{\mathrm{a}}-i \frac{\partial \rho}{\partial \tau}=0$; by combining these outcomes, integrating then submitting the spatial rate in Eq. (10) to their outcome, the density evolution is obtained

$$
\rho_{\eta}=c_{\eta}{ }^{-6} D_{0}\left(1-\cos \frac{\eta}{r_{0}}\right)^{-3}
$$

where $D_{0}$ is a constant from the integration. According to Eq. (8), the Hubble parameter rate is depended on the density and by submitting Eq. (13) to it

$$
\int_{H_{0}}^{H} d H=\int_{\eta_{0}}^{\eta}-\frac{4 \pi G_{\alpha}}{3 c_{\eta}{ }^{6}} D_{0}\left(1-\cos \frac{\eta}{r_{0}}\right)^{-3} d \eta
$$

The expansion rate is obtained by performing the integrating with utilising Eq. (1) as

$$
H_{\eta}=\frac{c_{\eta}{ }^{-2}}{6 E_{D}} D_{0}\left(\frac{1}{5} \cot ^{5} \frac{\eta}{2 r_{0}}+\frac{2}{3} \cot ^{3} \frac{\eta}{2 r_{0}}+\cot \frac{\eta}{2 r_{0}}\right)+H_{0}
$$

where $H_{0}$ denotes the integration constant. Utilising Eqs. $(10,12-15)$ in the complex plane generates the polar radius of the complex spacetime worldlines

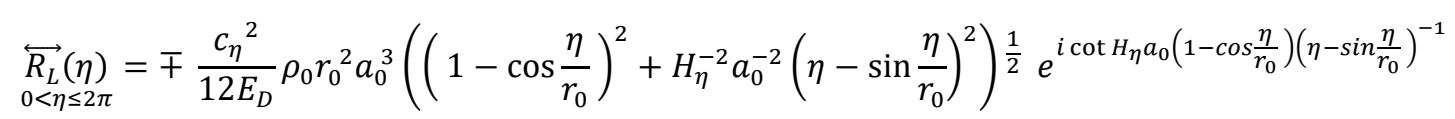

The $\mp$ solutions of the polar radius imply the matter and antimatter are evolving in opposite directions. The $G_{\eta}$ and $c_{\eta}$ are evolving along with the spacetime worldline direction and constant at perpendicular worldlines as will be discussed in the next section.

\section{EVOLUTION OF SPACETIME WORLDLINES}

By tuning the integration constants of the derived model in Eq. (16) to guide the mean evolution value of the Hubble parameter at $\sim 70 \mathrm{~km} \cdot \mathrm{s}^{-1} \cdot \mathrm{Mpc}^{-1}$ and a phase transition of expansion at universe age of $\sim 9 \mathrm{Gyr}$ as well as considering a positive initial curvature $r_{0}{ }^{2}=1.1$ based on the recent findings from CMB [4], the predicted density at the phase transition $\Omega_{t}=\sim 1.12$. In addition, the predicted spacetime worldlines of both sides are shown in Fig. 1, where the cosmic evolution is predicted to experience three distinct phases.

First, both sides of the universe expand away from the Bounce Bang, which could be due to the Bang and electromagnetic fields that separated matter and antimatter and propelled them in opposite directions, where they would be blue and red shifted in corroboration to the CMB dipole anisotropy. During the first phase (i.e., the first $\sim 9 \mathrm{Gyr}$ ), the gradients of the worldlines indicate the expansion is decelerating. The expansion rate is discussed in detail in Section 5. In the second phase by contrast, the worldlines reverse their directions, with both sides entering a state of free fall toward each other. It is conceivable that the matter and antimatter are free-falling toward each other at gravitational acceleration, causing the current accelerated expansion. The gradients of worldlines during this phase show an accelerated rate. These two phases can be explained using the analogy of a ball being thrown upwards, it decelerates until it stops, then it reverses its direction and free falls at accelerating rate. 
Interestingly, the model predicts a third phase of spatial contraction with a reversal-time arrow that appears after $\sim 18$ Gyr. In this phase, the universe experiences a contraction, which could be due to a high concentration of matter/antimatter in both sides, leading to the Big Crunch.

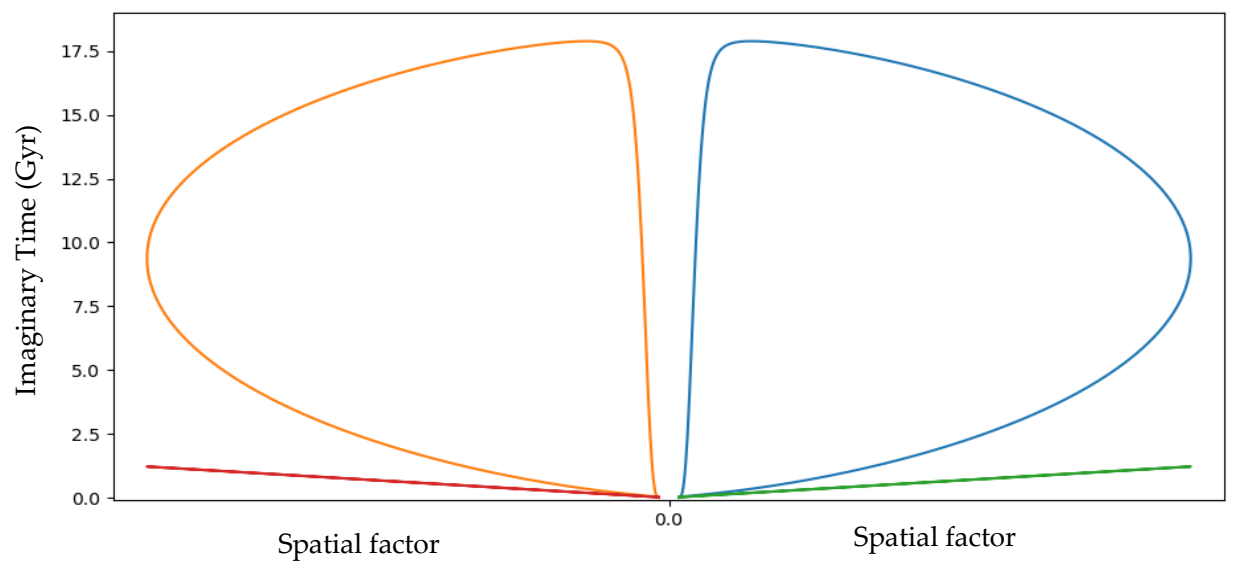

FIG. 1. Evolution of polar radius of complex spacetime worldlines of the spatial factor evolution through the imaginary time of both sides (curves) in addition to the straight lines of light worldlines (diagram is not to scale). The evolutions are, at first, a phase of spatial expansion away from the Bounce Bang of both sides. Then, a second spatial expansion phase in the reverse direction as both sides free-fall toward each other at gravitational acceleration. Finally, a third time-reversal phase of spatial contraction leading to the Big Crunch.

A 3D spatial and 1D temporal dimensions schematic representation of the spacetime worldline evolutions of both sides of the universe is shown in Fig. 2.

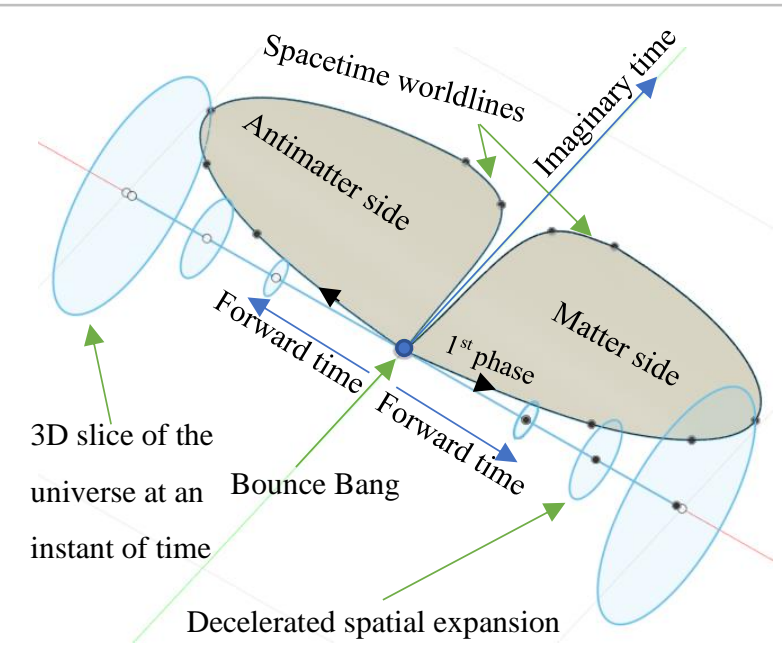

(a)

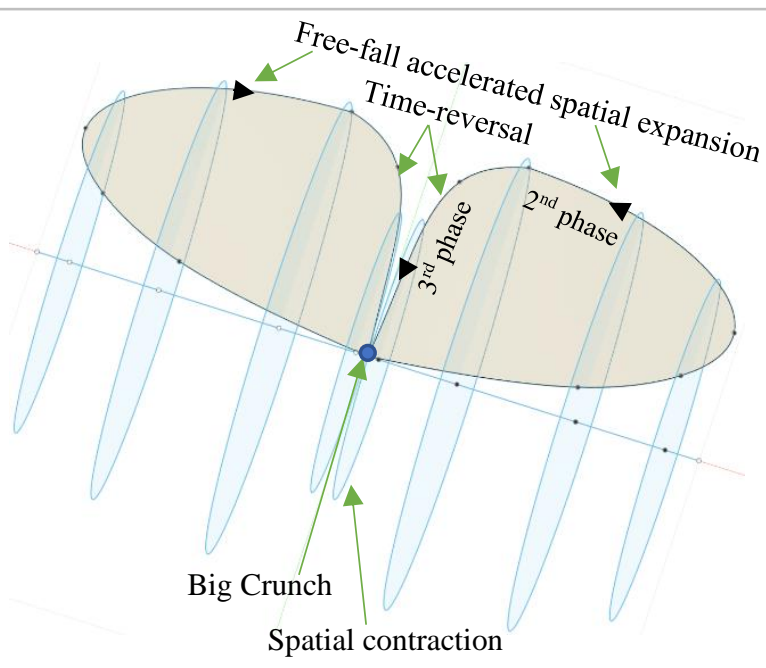

(b)

FIG. 2. Schematic representation in 3D spatial and 1D temporal dimensions of both sides according to the spacetime worldline evolution. (a) In the first phase, both sides expand away from the Bounce Bang. (b) In the second stage, both sides expand in reverse directions and free-fall toward each other at gravitational acceleration. In the third phase, both sides contract, due to high concentrations of matter/antimatter leading to the Big Crunch. Blue circles represent a 3D universe slice, which is not necessarily to be a simply path connected.

The $G_{\eta}$ and $c_{\eta}$ evolve along with spacetime worldlines and remain constant at the perpendicular directions. The $\tau c_{\eta}$ effects each side directly by their expansion. Forward time affects the expansion of both sides due to gravity between them, while the third time component may have lesser influence. The spacetime worldlines appear straight lines due to gravitational lensing as in the next section. 


\section{SIMULATION OF SPACETIME CURVATURE}

The spacetime worldlines of a single side can be simulated according to the derived model with nearby spacetime worldlines corresponding to the early and present universe, as shown in Fig. 3. In the early universe, simulation of spacetime worldline evolutions coupled with both flat and positive initial curvature are not equal at any age as shown in Fig. 3(a). This reveals that the spacetime is curved similar to a ball curved surface, which aligns with recent reports of space curvature from CMB [4]. Conversely, for the present accelerated phase of expansion in the reverse direction, simulation of the evolution of spacetime worldlines coupled with positive initial curvature produce a flat end or flat spacetime, as shown in Fig. 3(b).

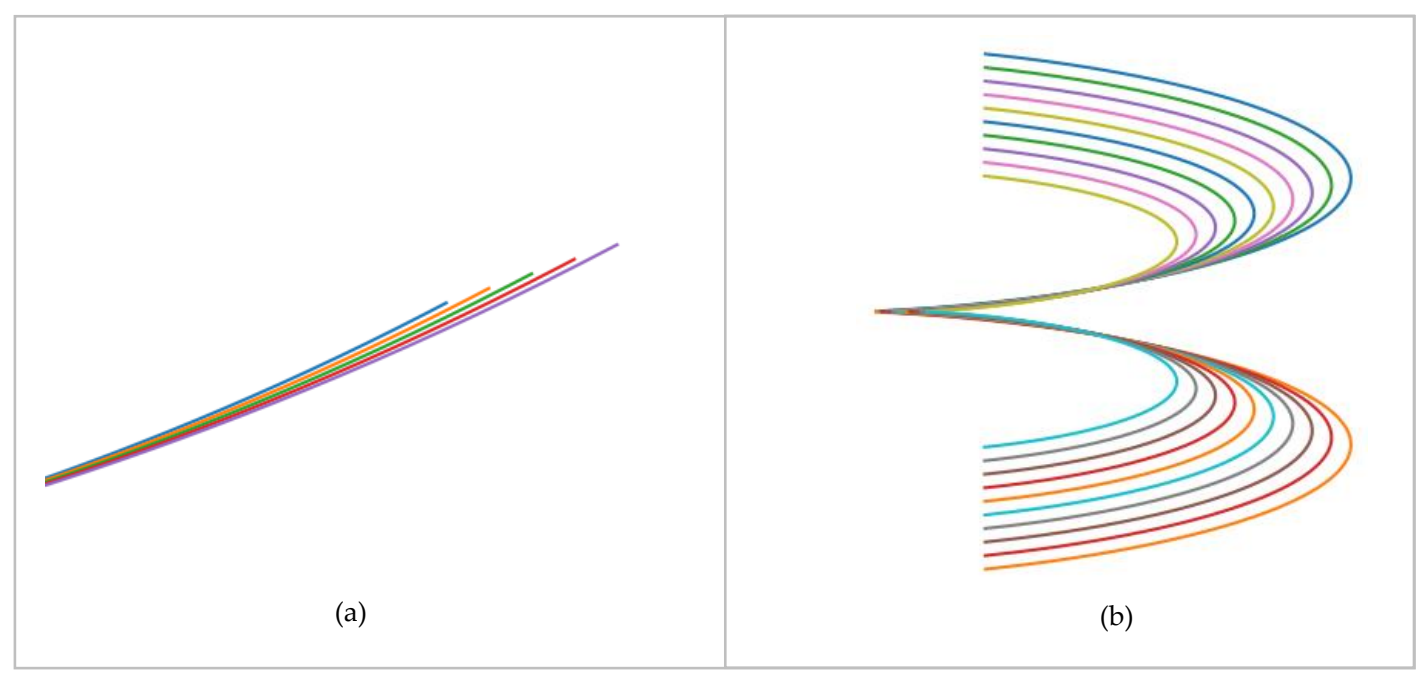

FIG. 3. Evolution of spacetime worldlines corresponding to (a) the early and (b) the present universe.

A schematic representation of 2D spatial and 1D temporal dimensions is shown in Fig. 4(a), while Fig. 4(b) shows an approximate apparent topology due to gravitational lensing.

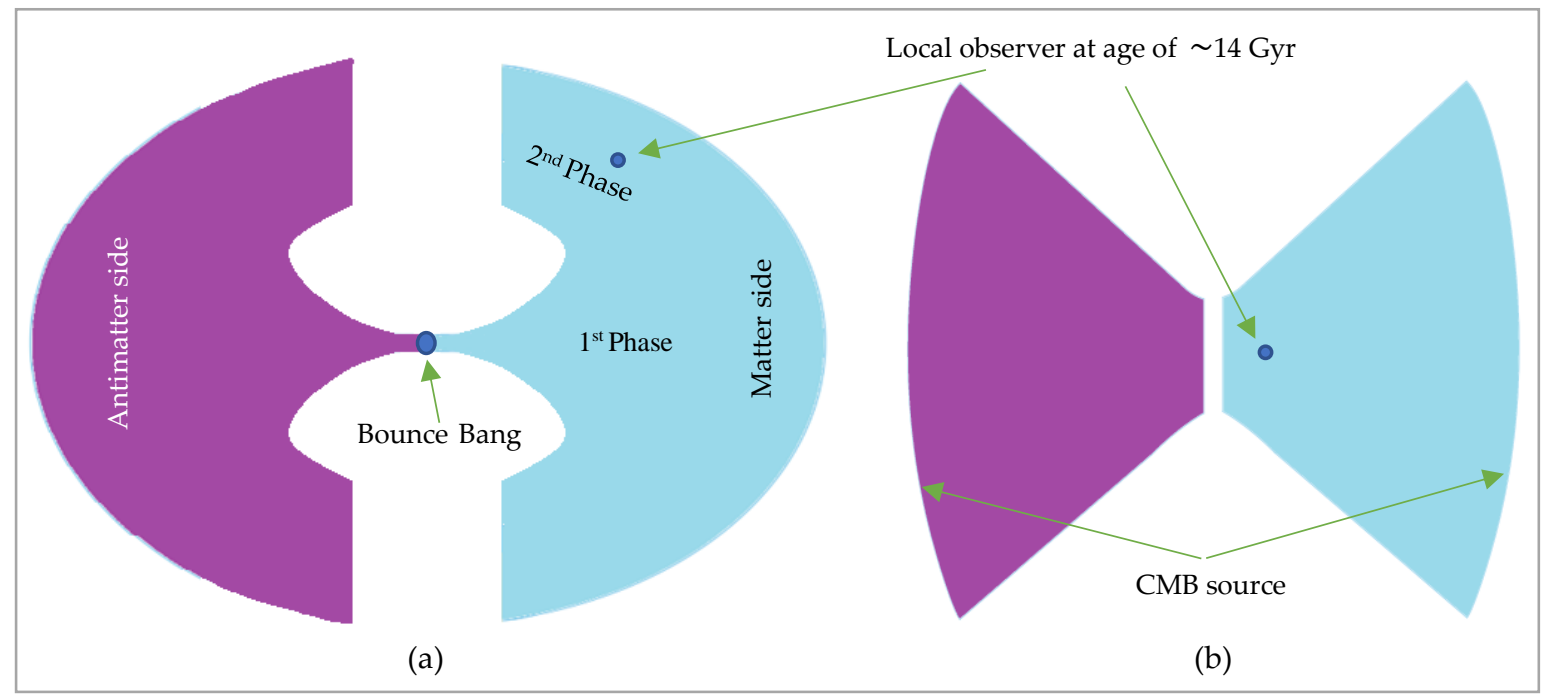

FIG. 4. (a) 2D-schematic representation of the predicted cosmic topology of both sides at the first phase away from the Bounce Bang; then the second phase corresponding to the reversal of the expansion direction. (b) The apparent topology during the first and second phases caused by the effects of gravitational lensing. 


\section{EXPANSION AND FLOW RATES}

\subsection{Evolution of the Hubble Parameter}

The Hubble parameter, or the speed of the spatial expansion $H \equiv \dot{a} / a$, and the deceleration/ acceleration $\dot{H}(\eta) \equiv \ddot{a} / a$ can be determined using Eqs. (15) and (13 in 8), respectively. The predicted speed and deceleration/acceleration of the spatial expansion are shown in Fig. 5.

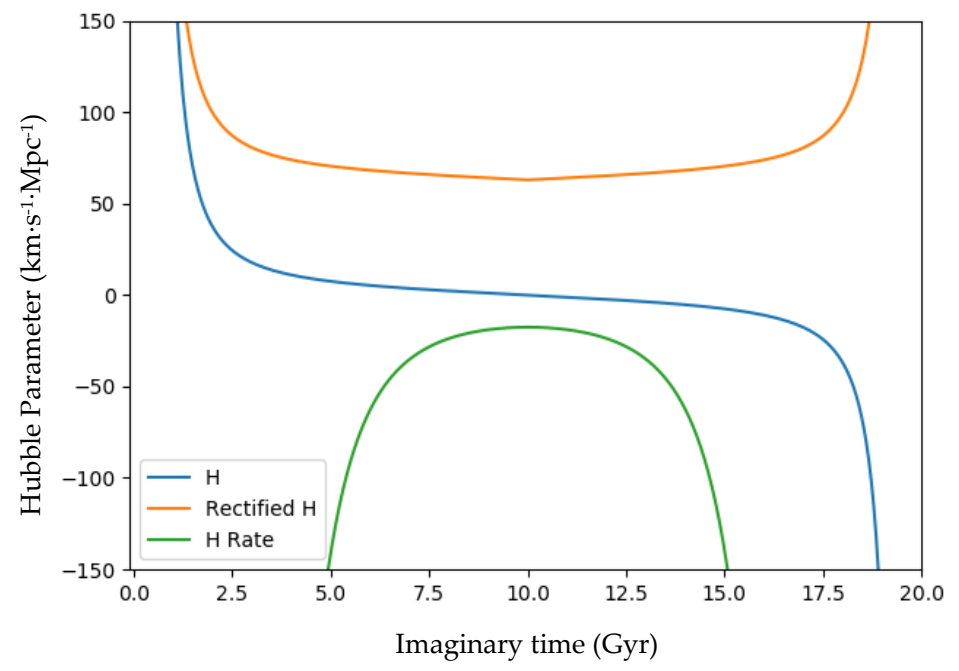

FIG. 5. Hubble parameter or the speed of the spatial expansion and the deceleration/acceleration.

The Hubble parameter shown in the blue curve starts at its highest value at the Bounce Bang, perhaps owing to the Bang where the speed of expansion is at its highest value. Then, the speed of expansion decreases during the first $\sim 9 \mathrm{Gyr}$, which could be due to gravity, until it approaches its minimal value at the phase transition at $\sim 9$ Gyr. Afterwards, the Hubble parameter starts to increase in the reverse direction (the minus sign of the speed at the second phase indicates the opposite direction, as discussed in the previous sections), which could be the result of both sides start to freefall toward each other. The parameter then reaches its zenith towards the Big Crunch.

According to the mechanics, the opposite signs of the acceleration (green curve) and the expansion speed in the first phase indicate a slowing down of the expansion rate, while the matching signs in the second phase indicate the expansion speed is increasing. A rectified Hubble parameter is shown in the orange curve to reflect the opposite direction of the expansion at the second phase where the integration constants were tuned to regulate the mean evolution value of the parameter at $\sim 70 \mathrm{~km} \cdot \mathrm{s}^{-1} \cdot \mathrm{Mpc}^{-1}$.

\subsection{Flow Rate of Both Sides}

Both sides are predicted to expand away from each other during the first phase, before they freefall or flow towards each other in the second phase according to Eq. (16). The flow rate/speed of the matter side of the universe due to the gravitational attraction by the antimatter side can be predicted by taking the derivative of the model in Eq. (15) regarding the imaginary conformal time

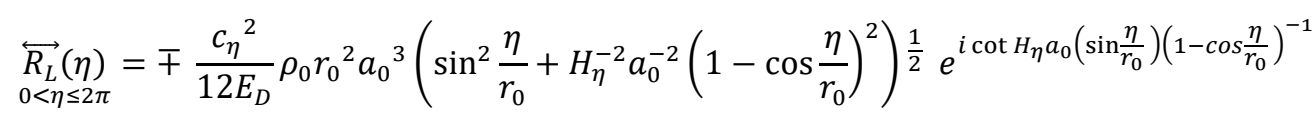




\section{SIMULATION OF SPIRAL GALAXY}

The consistent patterns of galactic rotation curves using precise and independent galactic redshift data have confirmed that hydrogen clouds and outermost stars are orbiting galaxies at speeds faster than those calculated using Newtonian laws. Accordingly, the dark matter hypothesis was introduced to account for the apparently missing galactic mass and to explain these fast-orbital velocities $[27,28]$. However, no evidence for the existence of dark matter, which is hypothesised to account for the majority of galactic mass, has been observed since its introduction. The failure to find dark matter led to the introduction of new theories such as modified gravity and modified Newtonian dynamics [29-33]. On the other hand, several recent studies report that many galaxies do not contain dark matter [34]. This scenario was used to inform galaxy formation simulations using modified Newtonian dynamics without considering the effects of dark matter [35]. Therefore, it seems that there is no evidence for (or agreement on) the existence or nature of dark matter, and it may be not an essential requirement for galaxy formation.

Alternatively, because the shapes of spiral galaxies are highly similar to vortex shapes, a spiral galaxy can be modelled as a forced vortex, where an external momentum is exerted on galaxies by the curvature of the spacetime along with its worldline evolutions. It is predicted by the model as shown in Fig. 1 and Fig. 4(a), the curvature of the spacetime increases along with its worldline evolution with the highest degree of curvature occurring at the phase transition. Accordingly, it can be inferred that the fast-orbital speeds observed for outer stars results from the variation of the spacetime curvature along with its worldline evolution.

To evaluate this, a fluid simulation study was performed based on Newtonian dynamics using the Fluid Pressure and Flow software [36]. In this simulation, a perfect fluid of mass density $\rho$ and isotropic pressure $p$ was assumed to represent the spacetime continuum according the postulated spacetime duality, while the fluid particles were traced to study the divergence of fluid flux. Using these conditions, a fluid model was built to simulate the spacetime continuum flux for incrementally flatter worldline curvature as shown in Fig. 6.

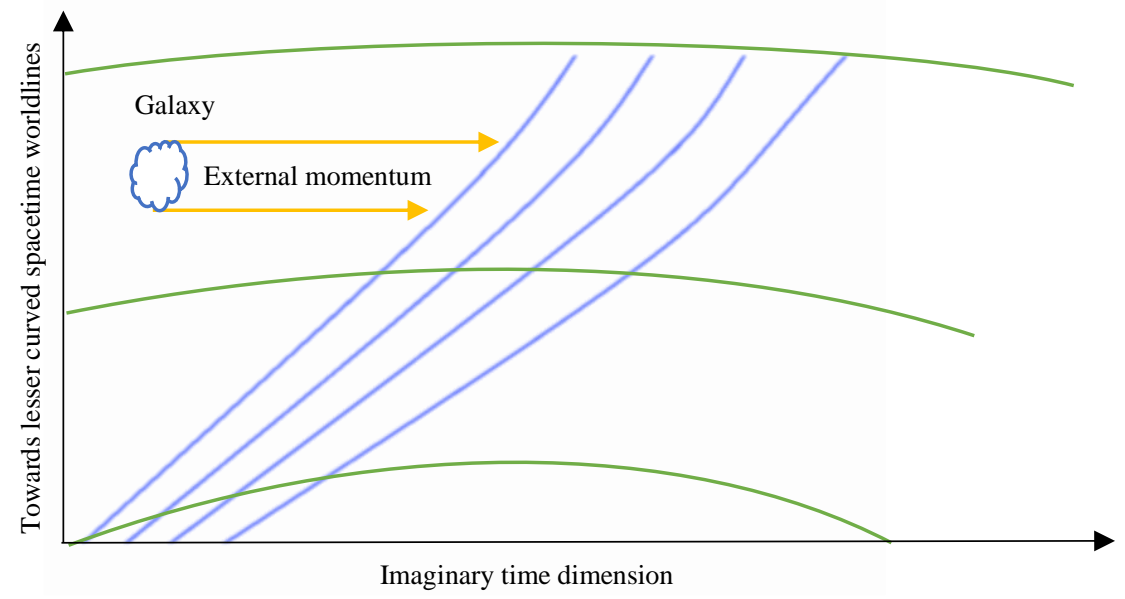

FIG. 6. External momentum exerted on a galaxy owing to the divergence of the spatial curvature through the imaginary time dimension along with the predicted spacetime worldlines. Green curves represent the curvature of spacetime worldlines. Blue curves represent the simulated spacetime continuum flux.

Using the resultant momentum, a simulation of a spiral galaxy as a forced vortex is shown in Fig. 7. 


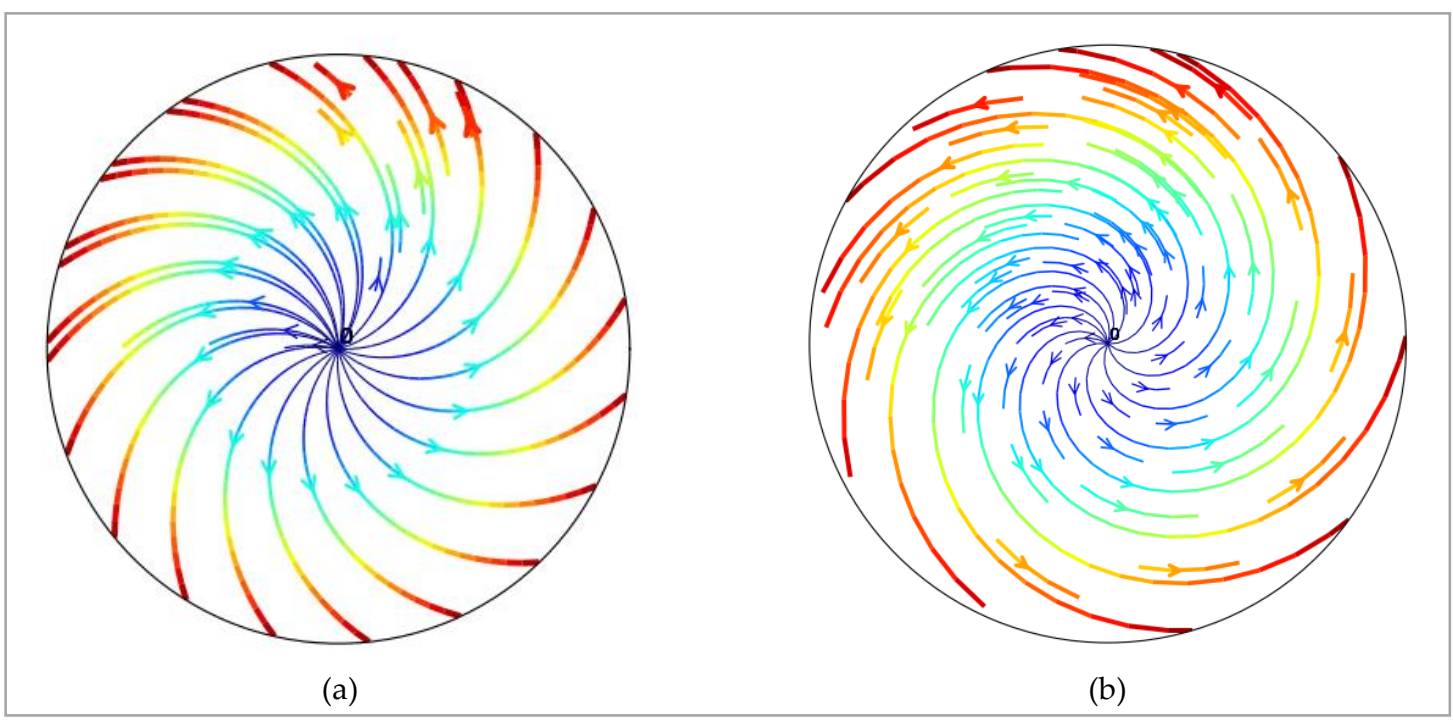

FIG. 7. Spiral galaxy rotation at (a) early and (b) present universe. Blue colour represents the slowest tangential speeds and red represents the fastest speeds.

The simulation shows the tangential speeds of outer parts of the spiral galaxy are faster in comparison with the speeds of inner parts. Additionally, the galaxies at the present universe are rotating faster than their speed at the early universe for the same mass. These results seem to follow the Tully-Fisher relation [37]. Based on the simulation results, it could be concluded that spacetime curvature along with its worldlines is responsible for the high speed of galaxies, explaining the dark matter.

\section{CONCLUSIONS}

In this study, the evolution of complex spacetime worldlines was utilised to construct a potential cosmic topology. The mathematical derivations of a positively curved universe governed by gravity alone revealed two opposite solutions of the worldline evolution, which imply that the universe has two sides: matter and antimatter. At the early universe, the matter would be blue shifted while antimatter would be redshifted in corroboration to the dipole of the CMB and anisotropy universe.

The derived model predicted a phase of decelerating spatial expansion during the first $\sim 9$ Gyr, followed by a second phase of accelerating expansion; potentially resolving the tension in the Hubble parameter measurements, with density $\Omega_{t}=\sim 1.12>1$ at the phase transition. Both sides of the universe expand away from the Bounce Bang at the first phase due to the Bang. Afterwards, at the second phase they reverse their directions and fall towards each other. It is conceivable that the matter and antimatter are free-falling toward each other at gravitational acceleration, causing the current accelerated expansion of the universe and explaining the dark energy. Additionally, the simulated curved spacetime during the decelerating phase was demonstrated to be flattened during the accelerating phase due to reverse direction of the worldlines, explaining the current space flatness.

Regarding the fast-orbital speed of stars, the simulation provided a physical explanation by which the spatial curvature through imaginary time dimension along with the spacetime worldlines of both sides was found to exert momentum on galaxies. Therefore, the geometrical spacetime curvature is causing them to increase in speed, which explains the dark matter.

Finally, the model predicted a final time-reversal phase of spatial contraction leading to the Big Crunch, thereby signifying a cyclic universe. 


\section{FUTURE WORK}

This study presented a new theory with promising outcomes and predictions, which can be verified, fine-tuned, or disproved using astronomical data in future works. The integration constants of this model can be fine-tuned to estimate the matter/antimatter and curvature densities accurately. Finally, the accurate age of the universe can be estimated based on the predicted Hubble parameter evolution rate.

\section{References}

[1] M. R. Wilczynska et al., "Four direct measurements of the fine-structure constant 13 billion years ago," Sci. Adv., vol. 6, no. 17, p. eaay9672, Apr. 2020.

[2] J. P. Uzan, "Varying constants, gravitation and cosmology," Living Reviews in Relativity, vol. 14, no. 2. Albert Einstein Institut, pp. 1-155, 29-Mar-2011.

[3] J. F. Donoghue, "Spatial and temporal gradients in the cosmological constant," J. High Energy Phys., vol. 7, no. 3, pp. 1115-1129, Mar. 2003.

[4] E. Di Valentino, A. Melchiorri, and J. Silk, "Planck evidence for a closed Universe and a possible crisis for cosmology," Nat. Astron., vol. 4, no. 2, pp. 196-203, Feb. 2020.

[5] A. TRAUTMAN, "Spin and Torsion May Avert Gravitational Singularities," Nat. Phys. Sci., vol. 242, no. 114, pp. 7-8, Mar. 1973.

[6] G. Unger and N. Popławski, "Big Bounce and Closed Universe from Spin and Torsion," Astrophys. Journal, 87078 (7pp), 2019, 2019.

[7] O. Klein, "Instead of cosmology," Nature, vol. 211, no. 5056. Nature Publishing Group, pp. 1337-1341, 1966.

[8] O. Klein, "Arguments concerning relativity and cosmology," Science (80-. )., vol. 171, no. 3969, pp. 339-345, Jan. 1971.

[9] P. J. E. Peebles and B. Ratra, "The cosmological constant and dark energy," Reviews of Modern Physics, vol. 75, no. 2. American Physical Society, pp. 559-606, 22-Apr-2003.

[10] E. Di Valentino, A. Melchiorri, and J. Silk, "Cosmic Discordance: Planck and luminosity distance data exclude LCDM," arXiv Prepr. arXiv2003.04935, Mar. 2020.

[11] N. Aghanim et al., "Planck 2018 results. V. CMB power spectra and likelihoods," Astron. Astrophys., vol. 89, Jun. 2020.

[12] M. Meneghetti et al., "An excess of small-scale gravitational lenses observed in galaxy clusters," Science (80-. )., vol. 369, no. 6509, pp. 1347-1351, Sep. 2020.

[13] S. Perlmutter et al., "Measurements of Omega and Lambda from 42 High-Redshift Supernovae," Astrophys. J., vol. 517, no. 2, pp. 565-586, Dec. 1998.

[14] A. G. Riess et al., "Observational Evidence from Supernovae for an Accelerating Universe and a Cosmological Constant," Astron. J., vol. 116, no. 3, pp. 1009-1038, Sep. 1998.

[15] A. G. Riess et al., "New Parallaxes of Galactic Cepheids from Spatially Scanning the Hubble Space Telescope: Implications for the Hubble Constant," Astrophys. J., vol. 855, no. 2, p. 136, Jan. 2018.

[16] A. G. Riess, "The expansion of the Universe is faster than expected," Nature Reviews Physics, vol. 2, no. 1. Springer Nature, pp. 10-12, 01-Jan-2020.

[17] K. Migkas, G. Schellenberger, T. H. Reiprich, F. Pacaud, M. E. Ramos-Ceja, and L. Lovisari, "Probing cosmic isotropy with a new X-ray galaxy cluster sample through the $\mathrm{L} X$ - T scaling 
relation," Astron. Astrophys., vol. 636, p. 15, Apr. 2020.

[18] E. Lusso et al., "Tension with the flat $\{\backslash$ Lambda $\} C D M$ model from a high redshift Hubble Diagram of supernovae, quasars and gamma-ray bursts," Astron. Astrophys., vol. 628, Jul. 2019.

[19] M. Al-Fadhli, "A new vision of the geometry of the cosmos as a twin-half closed Universe: accelerated expansion due to free fall gravitational attraction," NATASTRON-20012929 Lines 18-21 submited 1400 GMT, 13/1/2020.

[20] G. Efstathiou, "Is the low cosmic microwave background quadrupole a signature of spatial curvature?," 2003.

[21] R. J. Adler, B. Casey, and O. C. Jacob, "Vacuum catastrophe: An elementary exposition of the cosmological constant problem," Am. J. Phys., vol. 63, no. 7, pp. 620-626, Jul. 1995.

[22] N. Straumann, "General Relativity (Graduate Texts in Physics)," in Springer, Springer, 2013.

[23] M. Lachì Eze-Rey and J.-P. Luminet, “COSMIC TOPOLOGY,” arXivgr-qc/9605010v2 9 Jan 2003, 2003.

[24] G. F. R. Ellis and H. van Elst, “Cosmological models (Carg \’\{e\}se lectures 1998),” Dec. 1998.

[25] J. Fl, L. D. Landau, and E. Ml Lifshitz, “The Classical Theory of Fields Third Revised English Edition Course of Theoretical Physics," 1967.

[26] B. Ryden, Introduction to Cosmology. San Francisco, CA, USA: Addison Wesley, ISBN 0-80538912-1., 2006.

[27] P. D. Mannheim and D. Kazanas, "Exact vacuum solution to conformal Weyl gravity and galactic rotation curves," Astrophys. J., vol. 342, p. 635, Jul. 1989.

[28] Y. Sofue and V. Rubin, "Rotation Curves of Spiral Galaxies," Annu. Rev. Astron. Astrophys., vol. 39, no. 1, pp. 137-174, Oct. 2000.

[29] A. Maeder, "An alternative to the LCDM model: the case of scale invariance," Astrophys. J., vol. 834, no. 2, p. 194, Jan. 2017.

[30] M. M. Brouwer et al., "First test of Verlinde's theory of Emergent Gravity using Weak Gravitational Lensing measurements," Mon. Not. R. Astron. Soc., vol. 466, no. 3, pp. 2547-2559, Dec. 2016.

[31] E. A. Chadwick, T. F. Hodgkinson, and G. S. McDonald, "Gravitational theoretical development supporting MOND," Phys. Rev. D - Part. Fields, Gravit. Cosmol., vol. 88, no. 2, p. 024036, Jul. 2013.

[32] J. R. Van Meter, "Dark-matter-like solutions to Einstein's unified field equations," Phys. Rev. D, vol. 97, no. 4, p. 044018, Feb. 2018.

[33] M. Milgrom, "MOND in galaxy groups: A superior sample," Phys. Rev. D, vol. 99, no. 4, p. 044041, Feb. 2019.

[34] Q. Guo et al., "Further evidence for a population of dark-matter-deficient dwarf galaxies," Nat. Astron., vol. 4, no. 3, pp. 246-251, Jul. 2019.

[35] N. Wittenburg, P. Kroupa, and B. Famaey, "The formation of exponential disk galaxies in MOND," Astrophys. J., vol. 890, no. 2, p. 173, Feb. 2020.

[36] Sam Reid et al, "Fluid Pressure and Flow, PhET Interactive Simulations." University of Colorado, 2013.

[37] R. B. Tully and J. R. Fisher, "A new method of determining distances to galaxies," Astron. Astrophys., vol. 54, pp. 661-673, 1977.s 